\title{
On Bounded Posets Arising from Quantum Mechanical Measurements
}

\author{
Dietmar Dorninger ${ }^{1} \cdot$ Helmut Länger ${ }^{1}$
}

Received: 25 February 2016 / Accepted: 31 May 2016 / Published online: 22 June 2016

(C) The Author(s) 2016. This article is published with open access at Springerlink.com

\begin{abstract}
Let $S$ be a set of states of a physical system. The probabilities $p(s)$ of the occurrence of an event when the system is in different states $s \in S$ define a function from $S$ to $[0,1]$ called a numerical event or, more precisely, an $S$-probability. If one orders a set $P$ of $S$-probabilities in respect to the order of functions, further includes the constant functions 0 and 1 and defines $p^{\prime}=1-p$ for every $p \in P$, then one obtains a bounded poset of $S$-probabilities with an antitone involution. We study these posets in respect to various conditions about the existence of the sum of certain functions within the posets and derive properties from these conditions. In particular, questions of relations between different classes of $S$-probabilities arising this way are settled, algebraic representations are provided and the property that two $S$-probabilities commute is characterized which is essential for recognizing a classical physical system.
\end{abstract}

Keywords Poset with an antitone involution · Quantum measurement · Multidimensional probability $\cdot$ Boolean orthoposet $\cdot$ Orthomodularity $\cdot$ Commutativity

\section{Introduction}

A characteristic feature of measurements in quantum mechanics is that one only deals with probabilities.

Support of the research of the second author by the Austrian Science Fund (FWF), project I 1923-N25, and the Czech Science Foundation (GAČR), project 15-34697L, as well as by the project "Ordered structures for algebraic logic", supported by AKTION Austria - Czech Republic, project 75p11, is gratefully acknowledged.

Helmut Länger

helmut.laenger@tuwien.ac.at

1 Institute of Discrete Mathematics and Geometry, TU Wien, Wiedner Hauptstraße 8/104, 1040 Vienna, Austria 
Let $S$ be set of states of a physical system and $p(s)$ the probability of the occurrence of an event when the system is in state $s \in S$. Taking into account $p(s)$ for all $s \in S$ we obtain a function from $S$ to $[0,1]$, which is called a multidimensional probability or, more precisely, an $S$-probability, or sometimes, more generally, a numerical event (cf. [1, 2] and [10]).

$S$-probabilities are related to Mackey's approach to axiomatic quantum mechanics (cf. [9]). Mackey considers a function $p: \mathcal{O} \times \mathcal{S} \times \mathcal{B} \rightarrow[0,1]$ (where $\mathcal{O}$ denotes the set of observables, $\mathcal{S}$ the set of states and $\mathcal{B}$ the set of Borel sets of the real line) satisfying certain axioms. If one fixes some $(A, E) \in \mathcal{O} \times \mathcal{B}$ then one obtains a function $p(A, ., E): \mathcal{S} \rightarrow$ $[0,1]$ which assigns to each state $s \in \mathcal{S}$ the probability of the event that the measurement of $A$ lies in $E$.

A set $P$ of $S$-probabilities can be ordered in a natural way by the order $\leq$ of functions. Further, one may expect that with every $p(s)$ also the counter-probability $p^{\prime}(s):=1-p(s)$ will be in $P$, which we find formalized in the following (general)

Definition 1.1 An antitone involution on a poset $P$ is a mapping ' $: P \rightarrow P$ satisfying the following conditions:

- $\quad$ if $p, q \in P$ and $p \leq q$ then $p^{\prime} \geq q^{\prime}$;

- if $p \in P$ then $\left(p^{\prime}\right)^{\prime}=p$.

Taking into account further properties of quantum mechanical or classical physical systems, in this paper more features are added to a set $P$ of $S$-probabilities resulting in various classes of multidimensional probabilities. Our goal is to show how these classes are related, study their structures and give answers to the question whether two $S$-probabilities commute and, in the course of that, whether one might assume to deal with a classical physical system. A classical physical system can be identified by the fact that the poset of $S$-probabilities is a Boolean algebra (cf. [1]).

In particular, we will prove a representation theorem for a class of posets with an antitone involution analogous to the Theorem by Mączyński and Traczyk (cf. [10]) who characterized orthomodular posets with a full set of states by so-called algebras of $S$-probabilities. (For the definition see below.)

Algebras of $S$-probabilities and hence orthomodular posets with a full set of states have been thoroughly studied in respect to algebraic properties and physical interpretations (cf. [1-4] and [6-10]) and there are also some results about a generalization of these structures (cf. [5]), namely so-called generalized fields of events. (The definition will also be given below.)

In this paper the focus is on weakening and modifying the axioms of algebras of $S$ probabilities motivated by possible outcomes of experimental data which would not fit into the forementioned concepts.

\section{Classes of $S$-Probabilities}

We start with some algebraic notions and considerations.

Let $P$ be a poset with an antitone involution' ${ }^{\prime}$. If the infimum of two elements $p, q$ of $P$ exists we will denote it by $p \wedge q$, and if the supremum of $p, q \in P$ exists we will denote it by $p \vee q$. Moreover, elements $p, q$ of $P$ are called disjoint if $p \wedge q=0$ and orthogonal, denoted by $p \perp q$, if $p \leq q^{\prime}$. Further, $P$ is called Boolean if disjointness implies orthogonality. If $P$ 
is bounded, $p \in P$ is called complemented if $p \wedge p^{\prime}=0$, and $P$ is called complemented if all of its elements are complemented. If $p$ is complemented one can conclude that $p \vee p^{\prime}=1$.

Lemma 2.1 A bounded poset with an antitone involution is complemented if and only if orthogonality implies disjointness.

Proof Let $P$ be a bounded poset with an antitone involution and $p, q \in P$. If we assume $P$ to be complemented and $p \perp q$ then $p \leq q^{\prime}$ and $q^{\prime} \wedge q=0$ and hence $p \wedge q=0$. Conversely, if orthogonality implies disjointness then $p \wedge p^{\prime}=0$ since $p \perp p^{\prime}$.

An orthomodular poset is a complemented bounded poset $P$ in which the join of two orthogonal elements exists and in which $q=p \vee\left(q \wedge p^{\prime}\right)$ for all $p, q \in P$ with $p \leq q$. A state on an orthomodular poset $P$ is a mapping $s: P \rightarrow[0,1]$ satisfying the following conditions:

- $\quad s(0)=0$ and $s(1)=1$;

- if $p, q \in P$ and $p \perp q$ then $s(p \vee q)=s(p)+s(q)$.

A set $T$ of states on $P$ is called full if for $p, q \in P s(p) \leq s(q)$ for all $s \in T$ implies $p \leq q$.

Next we turn our attention to definitions motivated by the outcome of physical measurements.

Let $P$ be a set of multidimensional probabilities associated with an experiment. First we suppose that the functions 1 and 0 representing the constant probabilities 1 and 0 for every $s \in S$ are always among $P$. Then we will add some properties known from orthomodular structures (like Hilbert-space quantum mechanical systems) or Boolean algebras (which characterize classical physical systems).

Definition 2.2 A generalized field of S-probabilities (cf. [5]) is a subset $P$ of [0,1] ${ }^{S}$ satisfying the following conditions:

(P1) $0,1 \in P$;

(P2) if $p \in P$ then $p^{\prime}=1-p \in P$;

(P3) if $p, q \in P$ and $p \perp q$ then $p+q \in P$.

We will denote the class of generalized fields of $S$-probabilities by $\mathcal{G} \mathcal{F}$.

An algebra of $S$-probabilities (cf. [1] and [10]) is a subset $P$ of $[0,1]^{S}$ satisfying (P1), (P2) and (P4):

(P4) If $p, q, r \in P$ and $p \perp q \perp r \perp p$ then $p+q+r \in P$.

The class of algebras of $S$-probabilities will be denoted by $\mathcal{A L}$.

The members of $\mathcal{G} \mathcal{F}$ are bounded posets with an antitone involution. As for elementary properties of members of $\mathcal{G} \mathcal{F}$ cf. [5]. With Boolean algebras of events axiom (P3) is a translation of $A \subseteq B^{\prime}$ for events $A$ and the complement of $B$ into $p \leq 1-q$.

Obviously, $\mathcal{A L} \subseteq \mathcal{G} \mathcal{F}$.

Axiom (P4) is motivated by classical event fields for which pairwise orthogonality of a triple $A, B, C$ of events implies $A \subseteq B^{\prime} \cap C^{\prime}=(B \cup C)^{\prime}$, which in terms of functions means $p \leq 1-(q+r)$. 
As shown in [10], up to isomorphism the members of $\mathcal{A L}$ are exactly the orthomodular posets having a full set of states. These structures have been intensely studied, in particular from the point of quantum structures (cf. [1-10]).

It turns out that Definition 2.2 is somewhat too strong: No non-trivial element of a member of $\mathcal{A L}$ can be $\leq 1 / 2$ or $\geq 1 / 2$ for all $s \in S$. This gives rise to the following definitions:

Definition 2.3 A weakly structured poset of $S$-probabilities is a subset $P$ of $[0,1]^{S}$ satisfying (P1), (P2) and (P5):

(P5) If $p, q, r \in P, p \perp q \perp r$ and $p \wedge r=0$ then $p+q+r \leq 1$.

Let $\mathcal{W S}$ denote the class of weakly structured posets of $S$-probabilities.

It is evident that every member of $\mathcal{W S}$ is Boolean.

Now we assume that the addition of functions should be considered as a partial operation within a weakly structured poset of $S$-probabilities. This leads to

Definition 2.4 A structured poset of $S$-probabilities is a subset $P$ of $[0,1]^{S}$ satisfying $(\mathrm{P} 1)$, (P2) and (P6):

(P6) If $p, q, r \in P, p \perp q \perp r$ and $p \wedge r=0$ then $p+q+r \in P$.

Let $\mathcal{S P}$ denote the class of structured posets of $S$-probabilities.

Obviously, $\mathcal{S P} \subseteq \mathcal{G} \mathcal{F} \cap \mathcal{W} \mathcal{S}$

Remark $2.5 \mathcal{S P} \varsubsetneqq \mathcal{W S}$ since $P:=\{0,1 / 8,1 / 2,7 / 8,1\} \subseteq[0,1]^{\{1\}}$ belongs to $\mathcal{W S} \backslash \mathcal{S P}$. This can be seen as follows: If $p, q, r \in P, p \perp q \perp r$ and $p \wedge r=0$ then $0 \in\{p, r\}$ and hence $p+q+r \in\{q+r, p+q\}$ which shows $p+q+r \leq 1$, i.e. $P \in \mathcal{W S}$. On the other hand, $0 \perp 1 / 8 \perp 1 / 2$ and $0 \wedge 1 / 2=0$, but $0+1 / 8+1 / 2=5 / 8 \notin P$ proving $P \notin \mathcal{S P}$.

\section{Lemma 2.6}

(i) A member of $\mathcal{S P}$ belongs to $\mathcal{A L}$ if and only if it is complemented.

(ii) A member of $\mathcal{A L}$ belongs to $\mathcal{S P}$ if and only if it is Boolean.

\section{Proof}

(i) Let $P \in \mathcal{S P}$. If $P \in \mathcal{A L}$ then it is complemented because, as mentioned above, every member of $\mathcal{A L}$ is an orthomodular poset. If, conversely, $P$ is complemented, $p, q, r \in P$ and $p \perp q \perp r \perp p$ then $r \leq p^{\prime}$ and $p^{\prime} \wedge p=0$ imply $r \wedge p=0$ and hence (P6) implies $p+q+r \in P$ showing $P \in \mathcal{A L}$.

(ii) Let $P \in \mathcal{A L}$. If $P \in \mathcal{S P}$ then it is Boolean. If, conversely, $P$ is Boolean then (P6) follows from (P4) from which we infer $P \in \mathcal{S P}$.

Example 2.7 For every integer $n>1$ the subset $B_{n}:=\{0,1 / n, 2 / n, \ldots, 1\}$ of $[0,1]^{\{1\}}$ belongs to $\mathcal{S P} \backslash \mathcal{A L}$. We have:

- $k / n \wedge m / n=0$ if and only if $0 \in\{k, m\}$;

- $k / n \perp m / n$ if and only if $k+m \leq n$. 
Example 2.8 For positive integers $n$ and $k$ with $k \mid n$ put $A_{n k}:=\left\{I_{A}|A \subseteq N, k||A|\right\}$, where $N:=\{1, \ldots, n\}$ and $I_{A}$ denotes the function from $N$ to $[0,1]$ defined by $I_{A}(x):=1$ if $x \in A$ and $I_{A}(x):=0$ if $x \in N \backslash A$. Then $A_{n k} \in \mathcal{A L}$. Moreover, $A_{n k} \in \mathcal{S P}$ if and only if $k \in\{1, n\}$.

Proof We have

- $\quad I_{A} \wedge I_{B}=0$ if and only if $|A \cap B|<k$;

- $\quad I_{A} \perp I_{B}$ if and only if $A \cap B=\emptyset$.

Making use of Lemma 2.6 we obtain: If $k \in\{1, n\}$ then $A_{n k}$ is Boolean. If $1<k<n$ then (P5) is violated by $I_{\{1, \ldots, k\}}, I_{\emptyset}$ and $I_{\{2, \ldots, k+1\}}$ and hence $A_{n k} \notin \mathcal{W S}$ and therefore $A_{n k} \notin \mathcal{S P}$ in this case.

\section{Theorem 2.9}

- $\quad \mathcal{A L} \cap \mathcal{S P}$ consists of all Boolean members of $\mathcal{A L}$.

- $\quad \mathcal{A L} \backslash \mathcal{S P}$ consists of all non-Boolean members of $\mathcal{A L}$.

- $\quad \mathcal{S P} \cap \mathcal{A L}$ consists of all complemented members of $\mathcal{S P}$.

- $\quad \mathcal{S P} \backslash \mathcal{A L}$ consists of all non-complemented members of $\mathcal{S P}$.

Hence, the algebras of S-probabilities which are Boolean are exactly the structured posets of $S$-probabilities which are complemented. Moreover, every structured poset of $S$-probabilities assuming only the values 0 and 1 belongs to $\mathcal{A L}$.

Proof This follows from Lemma 2.6 and the fact that structured posets of $S$-probabilities assuming only the values 0 and 1 are complemented.

As already pointed out, members of $\mathcal{A L}$ are orthomodular posets admitting a full set of states. Since every arbitrary Boolean orthomodular poset admits a full set of states (cf. [10]) all Boolean orthomodular posets and in particular all Boolean algebras are among the posets representing members of $\mathcal{A L}$. (For a characterization of those posets by properties of $S$-probabilities cf. [1, 7] and [10]).

All sets of $S$-probabilities specified in Theorem 2.9 give rise to interpretations as quantum structures. If such a quantum structure turns out to be a Boolean algebra this will indicate that one deals with a classical situation (cf. e.g. [1]).

\section{An Algebraic Representation of Weakly Structured Posets of $S$-Probabilities}

We start by extending the notion of states to a class of bounded posets with an antitone involution.

Definition 3.1 A pseudostate on a bounded poset $P$ with an antitone involution in which the join of two disjoint elements exists is a mapping $s: P \rightarrow[0,1]$ satisfying the following conditions:

- $\quad s(0)=0$ and $s(1)=1$;

- $\quad$ if $p \in P$ then $s\left(p^{\prime}\right)=1-s(p)$;

- $\quad$ if $p, q \in P$ and $p \leq q$ then $s(p) \leq s(q)$;

- if $p, q \in P$ and $p \wedge q=0$ then $s(p \vee q)=s(p)+s(q)$. 
A set $T$ of pseudostates on $P$ is called full if for $p, q \in P, s(p) \leq s(q)$ for all $s \in T$ implies $p \leq q$.

We first prove a lemma which will often be used in the sequel.

Lemma 3.2 Assume $p, q \in P \in \mathcal{W S}$ and $p \wedge q=0$. Then $p+q=p \vee q$.

Proof If $r \in P$ is an upper bound of $p$ and $q$ then $p \perp r^{\prime} \perp q$ and hence $p+q+r^{\prime} \leq 1$ according to (P5), i.e. $p+q \leq r$. Together with $p, q \leq p+q$ this shows $p+q=p \vee q$.

Theorem 3.3 Up to isomorphism, the members of $\mathcal{W S}$ are exactly the bounded posets with an antitone involution in which the join of two disjoint elements exists and which have a full set of pseudostates.

Proof Let $P \in \mathcal{W S}$ with $P \subseteq[0,1]^{S}$. Then clearly $P$ is a bounded poset with an antitone involution.

Now we define $s_{x}(p):=p(x)$ for all $x \in S$ and $p \in P$.

For $a \in S$ and $p, q \in P$ we have $s_{a}(0)=0(a)=0, s_{a}(1)=1(a)=1, s_{a}\left(p^{\prime}\right)=p^{\prime}(a)=$ $1-p(a)=1-s_{a}(p)$ and in case $p \leq q, s_{a}(p)=p(a) \leq q(a)=s_{a}(q)$. Moreover, if $p \wedge q=0$ then

$$
s_{a}(p \vee q)=s_{a}(p+q)=(p+q)(a)=p(a)+q(a)=s_{a}(p)+s_{a}(q) .
$$

If $s_{x}(p) \leq s_{x}(q)$ for all $x \in S$ then $p \leq q$, proving that $\left\{s_{x} \mid x \in S\right\}$ is a full set of pseudostates on $P$. Hence $P$ is a bounded poset with an antitone involution in which the join of two disjoint elements exists and which has a full set of pseudostates.

Conversely, let $P$ be a bounded poset with an antitone involution in which the join of two disjoint elements exists and which has a full set $S$ of pseudostates. We define a mapping $f: P \rightarrow[0,1]^{S}$ by $(f(p))(s):=s(p)$ for all $p \in P$ and all $s \in S$. Then we have for arbitrary $p, q \in P$ :

- $\quad(f(0))(s)=s(0)=0$ for all $s \in S$ and hence $f(0)=0$.

- $\quad(f(1))(s)=s(1)=1$ for all $s \in S$ and hence $f(1)=1$.

- $\left(f\left(p^{\prime}\right)\right)(s)=s\left(p^{\prime}\right)=1-s(p)=1-(f(p))(s)=(f(p))^{\prime}(s)$ for all $s \in S$ and hence $f\left(p^{\prime}\right)=(f(p))^{\prime}$.

- The following are equivalent: $f(p) \leq f(q),(f(p))(s) \leq(f(q))(s)$ for all $s \in S$, $s(p) \leq s(q)$ for all $s \in S, p \leq q$.

From the last two properties of $f$ it follows for arbitrary $p, q \in P$ :

- $f(p)=f(q)$ if and only if $p=q$;

- $f(p) \perp f(q)$ if and only if $p \perp q$.

Now we prove $f(P) \in \mathcal{W S}$.

(P1) $0=f(0) \in f(P)$ and $1=f(1) \in f(P)$.

(P2) If $p \in P$ then $(f(p))^{\prime}=f\left(p^{\prime}\right) \in f(P)$.

(P5) Assume $p, q, r, t \in P, f(p) \perp f(q) \perp f(r)$ and $f(p) \wedge f(r)=f(0)$. Then $p \perp q \perp r$. If $t \leq p, r$ then $f(t) \leq f(p), f(r)$, from which we infer $f(t)=f(0)$, i.e. 
$t=0$, showing $p \wedge r=0$. Hence $p \vee r$ exists. Since $p, r \leq q^{\prime}$ we have $p \vee r \leq q^{\prime}$ and therefore

$$
\begin{aligned}
(f(p)+f(r))(s) & =(f(p))(s)+(f(r))(s)=s(p)+s(r)=s(p \vee r)= \\
& =(f(p \vee r))(s) \leq\left(f\left(q^{\prime}\right)\right)(s)=(f(q))^{\prime}(s)=1-(f(q))(s)
\end{aligned}
$$

for all $s \in S$, i.e. $f(p)+f(r) \leq 1-f(q)$ which is equivalent to $f(p)+f(q)+f(r) \leq 1$.

So $f(P) \in \mathcal{W S}$ and $f$ is an isomorphism from $P$ onto $f(P)$. Hence $P$ is isomorphic to a member of $\mathcal{W S}$.

This theorem is analogous to the theorem that up to isomorphism the algebras of $S$ probabilities are exactly the orthomodular posets having a full set of states.

\section{Characterizing Lattices and Boolean Algebras Among Structured Posets of $S$-Probabilities}

Having in mind that two disjoint elements of a member of $\mathcal{W S}$ and hence of $\mathcal{S P}$ have a join which is their sum (cf. Lemma 3.2) the question arises under which circumstances members of $\mathcal{S P}$ are lattices, all the more as the classicality of a physical system will correspond to members of $\mathcal{S P}$ that are Boolean lattices.

As for the example of $A_{n k}$ (Example 2.8) we have

Lemma 4.1 $A_{n k}$ is a lattice if and only if $k \in\{1, n / 2, n\}$ and hence every $A_{n k}$ belonging to $\mathcal{S P}$ is a lattice.

Proof It is easy to see that $A_{n k}$ is a lattice if $k \in\{1, n / 2, n\}$. Otherwise, $I_{\{1, \ldots, k\}} \vee I_{\{2, \ldots, k+1\}}$ does not exist.

Assume $p, q \in P \in \mathcal{S P}$. We point out that $p+q=p \vee q$ in case $p \wedge q=0$ (cf. Lemma 3.2). Further, the De Morgan laws hold if the respective join or meet exists (since' is an antitone involution). Moreover, it is easy to see that if one of $p$ and $q$ is complemented then $p \perp q$ implies $p \wedge q=0$. So $p \perp q$ is equivalent to $p \wedge q=0$ in case one of $p$ and $q$ is complemented. Finally, if one of $p$ and $q$ is complemented and $p \geq q$ then $p-q=p \wedge q^{\prime}$. This can be seen as follows: One of $p^{\prime}$ and $q$ is complemented and $p^{\prime} \perp q$ which implies $p^{\prime} \wedge q=0$, showing that $p^{\prime} \vee q$ exists and $p^{\prime} \vee q=p^{\prime}+q$. Therefore $p \wedge q^{\prime}$ exists and $p \wedge q^{\prime}=\left(p^{\prime} \vee q\right)^{\prime}=\left(p^{\prime}+q\right)^{\prime}=1-(1-p+q)=p-q$.

As usual with orthomodular lattices we say for $p, q \in P$ that $p$ commutes with $q$ if $p \wedge q$, $p \wedge q^{\prime}$ and $(p \wedge q) \vee\left(p \wedge q^{\prime}\right)$ exist in $P$ and $(p \wedge q) \vee\left(p \wedge q^{\prime}\right)=p$.

Theorem 4.2 Let $P$ be a complemented member of $\mathcal{S P}$. Then the following hold:

(i) $P$ is an orthomodular lattice if and only if for all $p, q \in P$ there exists a unique $r \in P$ with $r \geq p, q$ and $r-p \leq r^{\prime}+q$.

(ii) $P$ is a Boolean algebra if and only if for all $p, q \in P$ there exists an $s \in P$ with $s \leq p \leq s+q \leq 1$. 
Proof By Lemma 2.6, $P \in \mathcal{A L}$.

(i) In [7] it was shown that $P$ is an orthomodular lattice if and only if for all $p, q \in P$ there exists a unique $r \in P$ with $r \geq p, q$ and $\left(r \wedge p^{\prime}\right) \wedge\left(r \wedge q^{\prime}\right)=0$. Now for $p, q, r \in P$ with $r \geq p, q$ the following are equivalent: $r-p \leq r^{\prime}+q, r \wedge p^{\prime} \leq\left(r \wedge q^{\prime}\right)^{\prime}$, $\left(r \wedge p^{\prime}\right) \perp\left(r \wedge q^{\prime}\right),\left(r \wedge p^{\prime}\right) \wedge\left(r \wedge q^{\prime}\right)=0$.

(ii) In [8] it was proved that $\{p, q\}$ generates a Boolean algebra if and only there exists an $s \in P$ with $s \leq p \leq s+q \leq 1$. If any two elements of $P$ generate a Boolean algebra then $P$ is a lattice and hence (since every member of $\mathcal{A L}$ is an orthomodular poset) an orthomodular lattice. It is well-known that an orthomodular lattice in which any two elements generate a Boolean algebra is itself a Boolean algebra.

Let $P \in \mathcal{S P}$ with $P \subseteq\{0,1\}^{S}$. Then, as stated in Lemma 2.6, $P \in \mathcal{A L}$ and, as shown in [6], $P$ is a so-called concrete quantum logic. For the reader's convenience we recall the definition of a concrete logic.

Definition 4.3 (cf. e.g. [11]) A concrete quantum logic is a subset $E$ of the power set of some set $M$ satisfying the following conditions:

- $\varnothing \in E$;

- if $A \in E$ then $A^{\prime}:=M \backslash A \in E$;

- if $A, B \in E$ and $A \cap B=\emptyset$ then $A \cup B \in E$.

$\left(E, \subseteq,^{\prime}, \emptyset, M\right)$ is an orthomodular poset.

From Theorem 4.2 we obtain the following

Corollary 4.4 Let $P \in \mathcal{S P}$ with $P \subseteq\{0,1\}^{S}$. Then

(i) $P$ is a lattice if and only if $\max (p, q) \in P$ for all $p, q \in P$.

(ii) $P$ is a Boolean algebra if and only if $\min \left(p, q^{\prime}\right) \in P$ for all $p, q \in P$.

Proof For $p, q, r, s \in P$ the assertion that $r \geq p, q$ and $r-p \leq r^{\prime}+q$ is equivalent to $r=\max (p, q)$ whereas $s \leq p \leq s+q \leq 1$ is equivalent to $s=\min \left(p, q^{\prime}\right)$.

A further possibility to detect whether one deals with a classical physical situation, i.e. with commuting $S$-probabilities, is to try to adapt a relevant result best-known for orthomodular lattices.

Theorem 4.5 For $P \in \mathcal{S P}$ the following hold:

(i) If $p, q \in P$ and there exist three pairwise disjoint complemented elements $x_{1}, x_{2}, x_{12}$ of $P$ with $x_{1}+x_{12}=p$ and $x_{2}+x_{12}=q$ then $p$ and $q$ commute with each other.

(ii) $P$ is a Boolean algebra if and only if it is complemented and for every $p, q \in P$ there exist pairwise disjoint $x_{1}, x_{2}, x_{12} \in P$ with $x_{1}+x_{12}=p$ and $x_{2}+x_{12}=q$.

Proof

(i) We start with pointing out that because $P$ is Boolean, $x_{1}, x_{2}$ and $x_{12}$ are pairwise orthogonal. Next we show $x_{12}=p \wedge q$. Obviously, $x_{12} \leq p, q$. If $s \in P$ and $s \leq p, q$ then $s \leq q=x_{2} \vee x_{12} \leq x_{1}^{\prime}$ from which we conclude $s \leq p \wedge x_{1}^{\prime}=\left(x_{1}+x_{12}\right)-x_{1}=$ 
$x_{12}$. We proceed by proving that $x_{1}=p \wedge q^{\prime}$. We have $x_{1} \leq p$ and $x_{1} \leq x_{2}^{\prime} \wedge x_{12}^{\prime}=$ $\left(x_{2} \vee x_{12}\right)^{\prime}=q^{\prime}$. If $t \in P$ and $t \leq p, q^{\prime}$ then $t \leq q^{\prime} \leq x_{12}^{\prime}$ and hence $t \leq p \wedge x_{12}^{\prime}=$ $\left(x_{1}+x_{12}\right)-x_{12}=x_{1}$. This way we obtain $p=x_{1} \vee x_{12}=\left(p \wedge q^{\prime}\right) \vee(p \wedge q)$ showing that $p$ commutes with $q$. A symmetry argument shows that $q$ also commutes with $p$.

(ii) The necessity of the condition becomes obvious if one defines for the elements $p$ and $q$ of a Boolean algebra $x_{1}:=p \wedge q^{\prime}, x_{12}:=p \wedge q$ and $x_{2}:=p^{\prime} \wedge q$. That it is also sufficient can be seen as follows: Assume the condition to hold. Then according to Lemma 2.6, $P \in \mathcal{A L}$ and, as shown in [1], if for every $p, q \in P \in \mathcal{A L}$ there exist pairwise orthogonal elements $x_{1}, x_{2}, x_{12}$ with $x_{1}+x_{12}=p$ and $x_{2}+x_{12}=q$, then $P$ is a Boolean algebra. Since $x_{1}, x_{2}, x_{12}$ are pairwise disjoint they are also pairwise orthogonal.

Theorem 4.5 is analogous to the well-known theorem characterizing Boolean algebras among orthomodular lattices and to the generalization of this theorem to orthomodular posets.

Acknowledgments Open access funding provided by TU Wien (TUW), Vienna, Austria.

Open Access This article is distributed under the terms of the Creative Commons Attribution 4.0 International License (http://creativecommons.org/licenses/by/4.0/), which permits unrestricted use, distribution, and reproduction in any medium, provided you give appropriate credit to the original author(s) and the source, provide a link to the Creative Commons license, and indicate if changes were made.

\section{References}

1. Beltrametti, E.G., Mạczyński, M.J.: On a characterization of classical and nonclassical probabilities. J. Math. Phys. 32, 1280-1286 (1991)

2. Chajda, I., Länger, H.: Spaces of abstract events. Intern. J. Theor. Phys. 52, 1818-1824 (2013)

3. Dorfer, G., Dorninger, D., Länger, H.: On algebras of multidimensional probabilities. Math. Slovaca 60, 571-582 (2010)

4. Dorfer, G., Dorninger, D., Länger, H.: On the structure of numerical event spaces. Kybernetica 46, 971981 (2010)

5. Dorninger, D.: On the structure of generalized fields of events. Contr. General Algebra 20, 29-34 (2012)

6. Dorninger, D., Länger, H.: On a characterization of physical systems by spaces of numerical events. ARGESIM Report 35, 601-607 (2009)

7. Dorninger, D., Länger, H.: Testing for classicality of a physical system. Intern. J. Theor. Phys. 52, 11411147 (2013)

8. Dorninger, D., Länger, H.: Probability measurements characterizing the classicality of a physical system. Rep. Math. Phys. 73, 127-135 (2014)

9. Mackey, G.W.: Mathematical Foundations of Quantum Mechanics. Dover, Mineola, New York (2004)

10. Mączyński, M.J., Traczyk, T.: A characterization of orthomodular partially ordered sets admitting a full set of states. Bull. Acad. Polon. Sci. Sér. Sci. Math. Astronom. Phys. 21, 3-8 (1973)

11. Pták, P.: Concrete quantum logics. Intern. J. Theor. Phys. 39, 827-837 (2000) 